\title{
Probing Local Vibration Modes at Single Planar Defects by Vibrational Spectroscopy
}

Xingxu Yan ${ }^{1}$, Chengyan Liu ${ }^{1}$, Chaitanya Gadre ${ }^{1}$, Lei Gu ${ }^{1}$, Toshihiro Aoki ${ }^{1}$, Tracy Lovejoy ${ }^{2}$, Niklas Dellby $^{2}$, Ondrej Krivanek ${ }^{2}$, Ruqian $\mathrm{Wu}^{1}$ and Xiaoqing $\mathrm{Pan}^{1}$

${ }^{1}$ University of California - Irvine, Irvine, California, United States, ${ }^{2}$ Nion Co., Kirkland, Washington, United States

Imperfections such as stacking faults and interfaces are recognized as decisive factors in modifying thermal properties and heat transport in crystal materials by scattering phonon and changing vibrational structure [1]. However, the effect of crystal defects on thermal conductivity is being theoretically treated by perturbation methods without taking into account the local change of phonon dispersion relation [2]. Direct observation of local vibrational structure at single crystal defect has not been realized until now. The main issue is the spatial resolution required to resolve individual defects is not achievable for most of phonondetecting techniques including optical, and inelastic X-ray and neutron spectroscopies [3]. Here we demonstrate that space- and angle-resolved vibrational spectroscopy in a transmission electron microscope (TEM) enables the study of the vibrational structure of individual crystal defects. At a single stacking fault in a cubic silicon carbide, the acoustic vibration modes at $\mathrm{X}$ point undergo a red shift of several millielectronvolts, become enhanced, and are confined to within a few nanometers of the stacking fault. The detection of interfacial modes at an atomically thin Si-Ge interface will be discussed as well [4]. Our work opens the door to investigating phonon propagation around crystal defects, and provides guidance to the engineering of desired thermal performance for semiconductor and power electronic devices.

Fig. 1(a) illustrates the change of spatial resolution $\left(d_{\mathrm{r}}\right)$, momentum resolution $\left(m_{\mathrm{r}}\right)$ and energy resolution $\left(E_{\mathrm{r}}\right)$ as a function of convergence semi-angle $(\alpha)$ using monochromated electron energy-loss spectroscopy (EELS) at $60 \mathrm{kV}$ in a Nion microscope. These curves facilitate the selection of an experimental setup for either spatially or momentum resolved spectroscopy. Fig. 1(b) displays a scanning transmission electron microscope (STEM) image of 3C-phase SiC with plenty of stacking faults. First, a convergence semiangle of $33 \mathrm{mrad}$ was chosen to provide atomic resolution imaging capability. Under this condition, high spatially resolved vibrational spectroscopy was performed with an energy resolution of $5.7 \mathrm{meV}$ [5]. The vibrational spectrum of defect-free $\mathrm{SiC}$ in Fig. 1(c) has four discernable peaks corresponding to longitudinal and transverse optical (LO/TO) and longitudinal and transverse acoustic (LA/TA) phonon modes respectively. Due to the angle-integrated condition, the energy values of these peaks showed disparity, compared to Raman spectroscopy and simulated phonon dispersion curves. Intriguingly, the vibrational spectrum at a single stacking fault exhibits remarkable signal enhancement of the TA mode at 30-50 meV. To disentangle the local variation of each phonon branch, momentum resolution was then introduced by decreasing the convergence semi-angle to $3 \mathrm{mrad}$ [6]. Vibrational spectra were collected at the $\mathrm{X}$ point, which is at the edge of first Brillouin zone (FBZ). The resulting vibrational spectrum of defectfree SiC in Fig. 1(d) comprises of the LO, TO, LA and TA modes and agrees with calculated phonon dispersion curves. The intensities of LA and TA modes are reduced and increased respectively at the stacking fault and can also be seen in the spectrum-image in Fig. 2(b). Fig. 2(c) indicates that the TA mode was red-shifted by $3.8 \mathrm{meV}$ at stacking fault. Both intensity modulation and associated energy red-shift are caused by the generation of a local defect phonon mode. This mode was located at lower energy than the original TA mode of $\mathrm{SiC}$ and possessed an optical-phonon-like behavior.

$\mathrm{SiC}$ is a wide-bandgap semiconductor and has been widely applied in electronic devices. The study of local vibrations at a stacking fault offers deep insight into understanding phonon-defect interactions and 
modulation of thermal properties for $\mathrm{SiC}$. A similar strategy was implemented in a Si-Ge interface. This result unveiled an interfacial mode at about $48 \mathrm{meV}$ previously not included in phonon dispersion curves of either Si or Ge [4]. Our method opens the possibility to study the local vibrational signal at structural and substitutional defects in materials. [7].
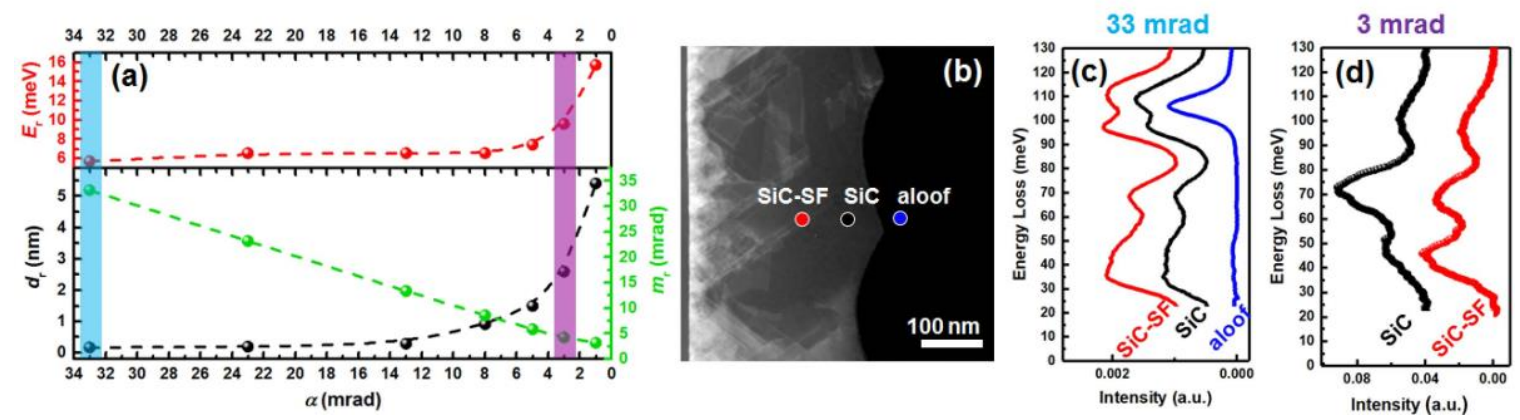

Figure 1. Vibrational spectra of defect-free $\mathrm{SiC}$ and stacking fault regions with wide and narrow momentum ranges. (a) The effect of probe convergence semi-angle $(\alpha)$ on the energy (Er), spatial (dr) and momentum (mr) resolutions at $60 \mathrm{kV}$. The dashed lines outline the general trend. (b) Low magnification STEM image of cross-sectional 3C-phase SiC grown on a Si substrate. (c) Vibrational spectra with $\alpha=33$ mrad of defect-free SiC, stacking fault, and aloof regions as marked in (b). (d) Angle-resolved vibrational spectra with $\alpha=3 \mathrm{mrad}$ of defect-free $\mathrm{SiC}$ and a stacking fault at $\mathrm{X}$ point.
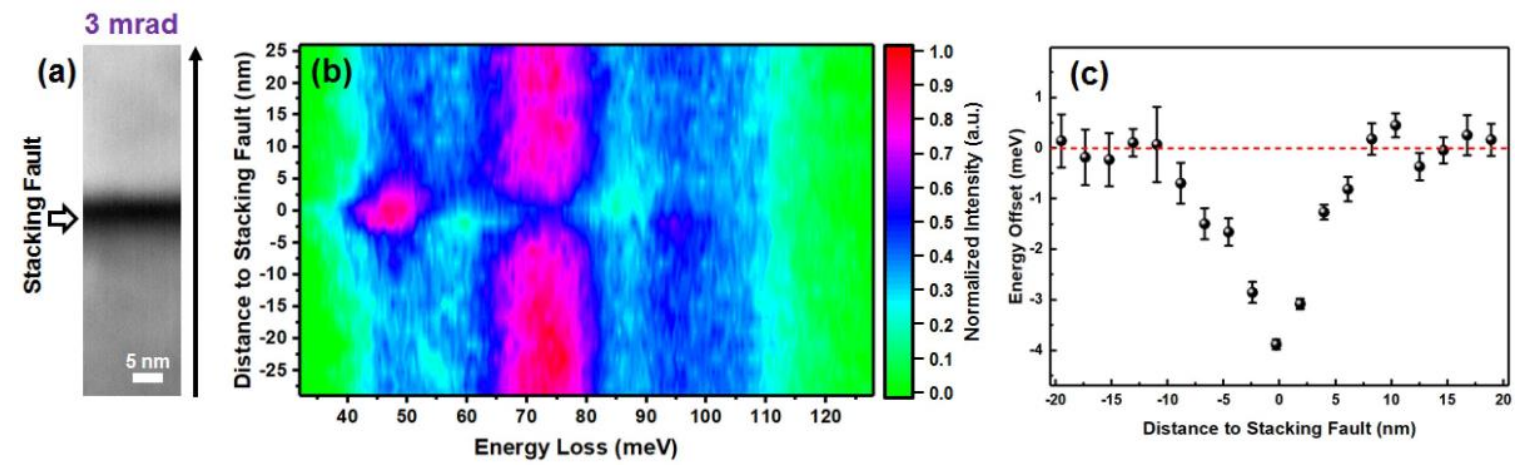

Figure 2. Spatial distribution of individual phonon modes and the red-shift of local phonon modes. (a) STEM image with $\alpha=3 \mathrm{mrad}$. (b) Line profile of the angle resolved vibrational spectra at X point across a stacking fault. (c) Energy offset of the TA peak as a function of location. Peak centers were determined by peak fitting of spectra in (b), and the energy value of TA mode (49 meV) in defect-free $\mathrm{SiC}$ was then subtracted to obtain the value of the energy offset.

\section{References}

[1] J. A. Krumhansl, J. Appl. Phys. 33 (1962), p. 307-319.

[2] M. D. Li et al. Nano Lett. 17 (2017), p. 1587-1594.

[3] O. L. Krivanek et al., Nature 514 (2014), p. 209-212.

[4] Y. Chalopin and S. Volz, Appl. Phys. Lett. 103 (2013), 051602.

[5] X. X. Yan et al., Nano Lett. 19 (2019), p. 7494-7502.

[6] F. S. Hage et al., Sci. Adv. 4 (2018), eaar7495.

[7] This work was supported by the Department of Energy (DOE) under Grant DE-SC0014430. TEM experiments were conducted using the facilities in the Irvine Materials Research Institute (IMRI) at the University of California - Irvine. 